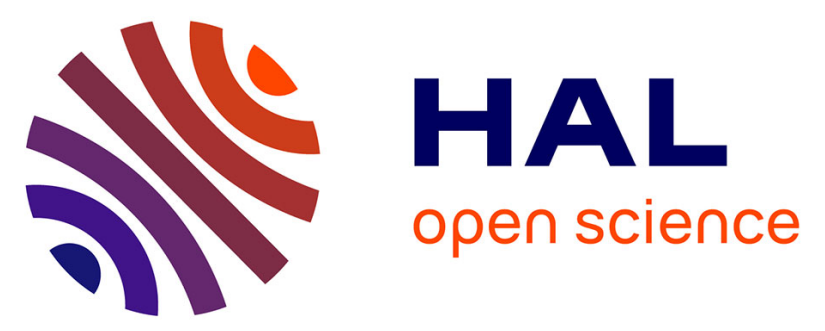

\title{
A quantitative study of track initialization of the four-frame best estimate algorithm for three-dimensional Lagrangian particle tracking
}

Alicia Clark, Nathanaël Machicoane, Alberto Aliseda

\section{- To cite this version:}

Alicia Clark, Nathanaël Machicoane, Alberto Aliseda. A quantitative study of track initialization of the four-frame best estimate algorithm for three-dimensional Lagrangian particle tracking. Measurement Science and Technology, 2019, 10.1088/1361-6501/ab0786 . hal-02519240

\author{
HAL Id: hal-02519240 \\ https://hal.science/hal-02519240
}

Submitted on 27 Mar 2020

HAL is a multi-disciplinary open access archive for the deposit and dissemination of scientific research documents, whether they are published or not. The documents may come from teaching and research institutions in France or abroad, or from public or private research centers.
L'archive ouverte pluridisciplinaire HAL, est destinée au dépôt et à la diffusion de documents scientifiques de niveau recherche, publiés ou non, émanant des établissements d'enseignement et de recherche français ou étrangers, des laboratoires publics ou privés. 


\title{
A quantitative study of track initialization of the Four-Frame Best Estimate algorithm for three-dimensional Lagrangian particle tracking
}

\author{
A. Clark, ${ }^{1}$ N. Machicoane ${ }^{1}$ and A. Aliseda ${ }^{1}$ \\ Department of Mechanical Engineering, University of Washington, Seattle, \\ Washington 98195-2600
}

(Dated: 8 February 2019)

We introduce a method to improve three-dimensional Particle Tracking Velocimetry (3D-PTV) algorithms. 3D-PTV is an experimental method used to measure the Lagrangian trajectories of individual particles over time. The trajectories are constructed by linking the particle positions from a sequence of images. Different 3DPTV algorithms have been proposed in the literature, ranging from simply taking the nearest neighbor in the next frame to using multiframe schemes. This work focuses on the initialization of the Four-Frame Best Estimate (4BE) method introduced by Ouellette et al. (2006), Experiments in Fluids, 40(2), 301-313. Previously, tracking algorithms have been initialized by using the particle's nearest neighbor(s) in the next frame or by using a velocity guess to predict the particle's location in the next frame. We propose a more robust initialization, coupled with 4BE, that performs better than existing methods in the literature, in the sense of yielding a higher number of correct tracks. The performance of the proposed initialization method is compared to the 4BE method that uses nearest neighbor initialization by applying both methods on direct numerical simulation data from the Johns Hopkins Turbulence Databases (JHTDB). We show that the modified initialization greatly improves tracking in two canonical cases, homogeneous isotropic turbulence and turbulent channel flow (inhomogenous and anisotropic), greatly increasing the percentage of correct tracks found even under challenging seeding/particle displacement conditions. 


\section{INTRODUCTION}

Three-Dimensional Particle Tracking Velocimetry (3D-PTV) is a Lagrangian method that is commonly used in fluid dynamic experiments to capture information about individual trajectories. The particles can be flow tracers if one is interested in characterizing the flow itself (e.g. turbulence dispersion, turbulence statistics, characteristic times and lengths) or disperse-phase particles if one wants to study the behavior of a second phase in a multiphase flow (e.g. particle slip velocity or acceleration, response time, and preferential concentration $)^{1,2}$. Additionally, tracking both flow tracers and disperse-phase particles, it is possible to discriminate the velocity fields for both phases to understand the underlying multiphase flow dynamics. The reconstructed particle trajectories allow for measurements of velocity and accelerations. If there are enough tracks captured, average quantities can be estimated on a grid, yielding Eulerian maps (3D3C velocity field) and other common Eulerian metrics, such as second order structure functions ${ }^{3}$.

Many 3D-PTV track-forming algorithms have been proposed in the literature ${ }^{4-22}$, and these methods vary based on the number of frames used to find particle trajectories (from 2 to $\mathrm{N}$ frames), the cost function used to assign particles to existing tracks, and the initialization methods used to create new tracks. The tracking scheme used for 3D-PTV is critical for accurate results. For example, if an incorrect particle position is assigned to a track in one time step, it not only creates a tracking error in that frame but can also propagate to subsequent frames and to other tracks: the incorrect particle assignment removes the particle from its true track, which can have two negative effects. First, the complete track will no longer be recovered. This can lead to errors in subsequent frames since the particles that were part of this track can now be assigned to other tracks. Second, this erroneous assignment also interrupts the track of the particle whose trajectory has been erroneously assigned. This can also lead to errors in subsequent frames since the future particle locations (that are part of the correct track that has been continued in error) can now be assigned to other tracks.

Another important consideration besides the tracking scheme itself is the initialization of the tracking scheme: the main focus of this article. The initialization method introduced here utilizes and extends the ideas proposed in some of the first 3D-PTV papers ${ }^{4-7,9-12,14}$, which used a search area for track initialization. These works used circular search areas for 
the initialization, but we will consider anisotropic search volumes that are adjustable in three different directions based on the flow characteristics being studied. For example, for a strong mean flow, the size of the search box can be adjusted based on the predicted flow velocity in the direction of the mean flow and can be smaller in the non-mean flow directions. This improves the accuracy of the tracking since it will limit the number of tracks started, which also aids in computational efficiency. Additionally, to our knowledge, there has not been a detailed analysis exploring the initialization of tracking algorithms, especially in different flow conditions.

Advanced 3D-PTV approaches, such as Shake-The-Box, have recently been developed and have shown great success at very high particle seeding densities ${ }^{23}$, allowing Eulerian interpolation to produce instantaneous 3D3C velocity fields. Such methods, however, require advanced equipment (high power lasers with repetition frequencies in the kilohertz, multiple cameras, and specialized software) and their use is non-trivial. Shake-The-Box methods have very significant computational costs and have complex set-up configurations. Thus, there is a continued need for accurate multiframe 3D-PTV algorithms to resolve turbulent flows in which seeding density and time-resolution do not require the added complexity of Shake-The-Box, but where current methods have either low accuracy or low yield of correct trajectories. The method proposed can be used with only one camera (capturing particles in a thick plane or using shadowgraphy), multiple cameras (illuminating a volume and resolving 3D flow or particle statistics), and is open-source. Additionally, the inputs to the proposed tracking algorithm depend solely on known flow characteristics (e.g. the expected maximum displacement of particles between frames). Consequently, the proposed tracking method performs extremely well in multiple simple applications such as dispersion in a turbulent channel flow. An example application is 2D tracking with one camera and backlighting, where the 3D physical space is projected onto the 2D sensor of the camera. The method performs extremely well in this application, even at higher particle seeding densities or in flows where there are large particle displacements between frames.

Malik et al. ${ }^{13}$ and Dracos ${ }^{15}$ introduced a four-frame particle tracking method for 3DPTV that minimizes changes in acceleration (4MA). Ouellette et al. ${ }^{19}$ then extended this algorithm and established a framework to evaluate the accuracy of four particle tracking algorithms. Each algorithm differs in the number of frames used to find the most likely position of the particle in the next step of the track, or on the cost function used to evaluate 
which one of the possible matches best extends the current track. Of the four different track assignment methods and cost functions explored, the Four-Frame Best Estimate method is shown to have superior performance in homogeneous isotropic turbulence. Each particle track is initialized by choosing the particle's nearest neighbor (in 3D space) in the next frame. The nearest neighbor initialization method works well when there is zero average flow velocity and when the particles displacement between frames is small compared to the interparticle distance. However, it starts to fail when the particles move a distance comparable to the minimum particle-particle distance between frames (trackability limit). When the distance that the particles travel between two frames is much longer than average interparticle distance, tracking is impossible without additional heuristics or detailed knowledge of the flow ${ }^{8,13,15,19}$. Additionally, its accuracy decreases drastically if there is an average flow that is inhomogeneous, as the mean flow can systematically bring other particles near the location of the original particle in the previous frame. If the mean flow is homogeneous or varies with time or space in a smooth and simple manner, the user can introduce a velocity guess for nearest-neighbor initialization to correct for this bias, but this method is prone to errors in the tracking when there is unsteady flow or velocity gradients, even for a simple canonical flow such as Poiseuille flow.

This paper focuses on extending the 4BE method because it has been used extensively in turbulence research since its introduction ${ }^{24-30}$, becoming a leading tool in turbulence and multiphase experimental research. Additionally, as the $4 \mathrm{BE}$ method is simply the $4 \mathrm{MA}$ algorithm with a modified cost function, the results of modifying the initialization of 4BE will also be applicable to 4MA tracking algorithms. To do so, we introduce a modified initialization coupled with the $4 \mathrm{BE}$ method to increase tracking performance and increase the "yield" of tracks as a percentage of possible tracks started by particles detected in the images, while maintaining the accuracy (percentage of correct tracks from the total number of tracks reported). The proposed modified initialization method uses a customizable search in frame $n+1$ to initialize multiple tracks from a single particle position in frame $n$. Each of these potential tracks are then followed through the next two frames $n+2$ and $n+3$, and the cost function for each potential track is minimized only after all possible trajectories for the original particle in frame $n$ have been considered through the three next frames. The geometry of the initialization search region and the maximum number of track candidates 
that are started from a single particle can be adjusted to adapt them to a large variety of flow conditions.

Two direct numerical simulation (DNS) datasets, forced homogenous isotropic turbulence and turbulent channel flow, were used to validate this new 3D-PTV initialization method and to compare its performance to the $4 \mathrm{BE}$ with nearest neighbor initialization. The forced isotropic turbulence dataset was used to explore tracking in a canonical setting where there is zero average velocity. In contrast, the turbulent channel flow dataset was used to look at tracking when there is a non-zero, inhomogeneous average flow. Tracking performance was then analyzed using previously introduced metrics ${ }^{13,19}$. For both datasets, we show that the modified method proposed reduces tracking error and increases the track number yield, with a moderate computational cost that is affordable with modern tools. Additionally, for the turbulent channel flow, the tracking error for the modified initialization method remains significantly smaller than the tracking error for the nearest neighbor initialization even for extreme values of the non-dimensional particle displacements between image frames.

The paper is organized as follows: the Four-Frame Best Estimate tracking algorithm and the modified method proposed here are introduced in Section II; the direct numerical simulation datasets used to validate the modified initialization method are briefly described in Section III 1; the results from the comparison between the initialization methods are then explored in Section III 2; and the conclusions from this study and potential for applications are summarized in Section IV.

\section{MODIFIED FOUR-FRAME BEST ESTIMATE LAGRANGIAN TRACKING METHOD WITH ENHANCED TRACK INITIALIZATION}

The simplest Particle Tracking Velocimetry technique, using the position of particles in two consecutive frames and choosing the nearest neighbor in frame $n+1$ as the most likely position of the particle in frame $n$, may lead to wrong matches when increasing the number

of particles in the field of view and/or the displacement of particles between frames. To overcome this limitation, multiframe particle tracking methods were developed (6-8,11,13-22 and references within). Malik et al. ${ }^{13}$ and Dracos ${ }^{15}$ introduced a four-frame particle tracking method that minimizes changes in acceleration (4MA). Ouellette et al. ${ }^{19}$ extended this algorithm by introducing a new cost function that minimizes the distance between a particle 
in the fourth frame and its estimated position in that frame; this method is known as the Four-Frame Best Estimate (4BE) method.

Briefly, the Four-Frame Best Estimate algorithm uses four-frames $(n, n+1, n+2$, and $n+3)$ to reconstruct particle trajectories, as illustrated in Fig. 1(a). Individual tracks are initialized by using the nearest neighbor method, which chooses as the second position in the track the particle that minimizes the distance between its location in frame $n+1$ and the original particle position in frame $n$. Once a track is started in this way, these first two locations in the track are used to predict the position $\tilde{x}_{i}^{n+2}$ of the particle in frame $n+2$ :

$$
\tilde{x}_{i}^{n+2}=x_{i}^{n+1}+\tilde{v}_{i}^{n+1} \Delta t
$$

where $x_{i}^{n+1}$ is the position of the particle in frame $n+1, \tilde{v}_{i}^{n+1}$ is the predicted velocity, and $\Delta t$ is the time between frames. A search region is then defined around this predicted location in frame $n+2$ to look for particles that are candidates to continue the track. The tolerance in the search is set to be as small as possible (usually a few pixels) since it is aimed at finding a single particle whose actual location in frame $n+2$ is closest to the prediction from frames $n$ and $n+1$. If no particle is found, the track is abandoned. If one particle is found, the track is continued with that particle. If more than one particle is found within this search region, each one can be used to predict a set of possible track continuations $\tilde{x}_{i}^{n+3}$ in frame $n+3$ :

$$
\tilde{x}_{i}^{n+3}=x_{i}^{n+1}+\tilde{v}_{i}^{n+1}(2 \Delta t)+\frac{1}{2} \tilde{a}_{i}^{n+1}(2 \Delta t)^{2}
$$

where $\tilde{a}_{i}^{n+1}$ is the predicted acceleration. A search region is defined around each of the $\tilde{x}_{i}^{n+3}$ possible track locations and actual particle locations found in those are used to extend the track candidates from frame $n+2$ to frame $n+3$. The $4 \mathrm{BE}$ algorithm chooses the most likely location of the particle and, therefore, the most likely track, by minimizing the cost function $\phi_{i j}^{n}$ :

$$
\phi_{i j}^{n}=\left\|x_{j}^{n+3}-\tilde{x}_{i}^{n+3}\right\|
$$

Equation 3 minimizes the distance between the actual particles $x_{j}^{n+3}$ and their predicted locations $\tilde{x}_{i}^{n+3}$. The track with the lowest cost represents the best candidate for track continuation. 
While 4BE with nearest neighbor initialization (4BE-NNI) is a very good compromise between low computational cost and tracking accuracy and efficiency, it discards many tracks that are incorrectly started by the nearest neighbor, thus prioritizing quick computational turn-around over the efficiency (the ratio of tracks detected to the total number of new particles where a track can be started). Additionally, there are certain cases where it can lose accuracy (the ratio of correct tracks to the total number of tracks completed). When the particle inter-frame displacement is comparable to the inter-particle distance for a significant number of particles (either because of high particle velocity fluctuations, or because of particle clustering reducing the inter-particle distance well below the median predicted from the volume fraction, or the combination of both), the percentage of bad tracks started with the nearest neighbor is high. These bad tracks will either be abandoned after frame $n+1$ (low efficiency) or be completed erroneously in frames $n+2$ and $n+3$ (low accuracy).

We have developed the Enhanced Track Initialization (ETI) to complement the strengths of the 4BE method and overcome its challenges, thereby extending its applicability to highly turbulent and inhomogeneous flows with high particle density, the traditional barrier between 3D-PTV methods and time-resolved high spatial resolution Shake-The-Box-PTV or PIV. Figure 1(b) highlights the features of the 4BE-ETI algorithm. This method uses multiple particle locations in frame $n+1$ (all particles found within the search region based on the estimated maximum particle displacements between two frames) to initialize, without prejudice for which one is more likely, multiple tracks. The shape and size of this initial search region is determined based on the flow characteristics. The initial dimensions of the search region were systematically determined using $110 \%$ of the estimated maximum particle displacement (in the $\mathrm{x}, \mathrm{y}$, and $\mathrm{z}$ directions) for each dataset. Typically, these maximum displacement values were different in each spatial direction, so the dimensions for the initial search region varied in each direction. The subsequent search region dimensions were determined using the mean displacement of the particles between frames. Both the initial and subsequent search regions were designed with a large safety factor, chosen to be conservative so that no tracks would be missed, while not increasing the computational time excessively. This allows the algorithm to explore multiple possible trajectories for each particle and eliminates the assumption that the closest particle in the next frame is the only option when starting a track. Subsequent search regions in frames $n+2$ and $n+3$, used for track continuation, are smaller since the continuation search uses a better estimate of local 
particle displacement, based on the velocity and acceleration estimates from the positions found in frames $n, n+1$, and $n+2$. This decreases computational costs because it limits the number of particles found, and therefore the number of potential tracks to follow, thus limiting possible track continuations.

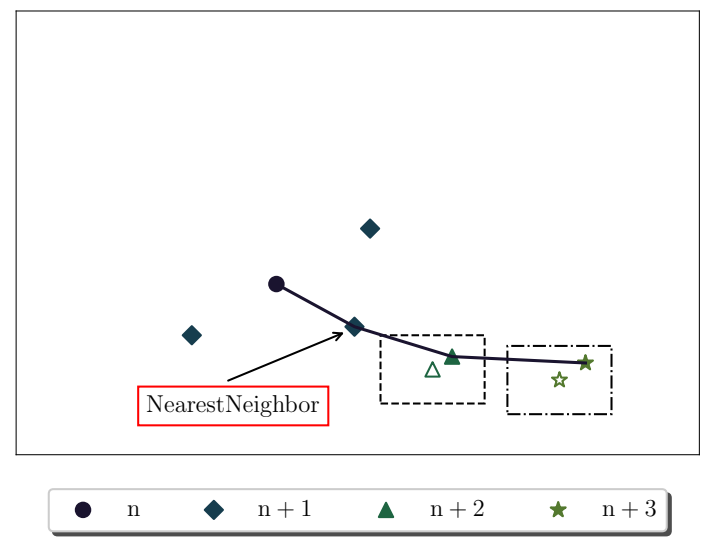

(a)

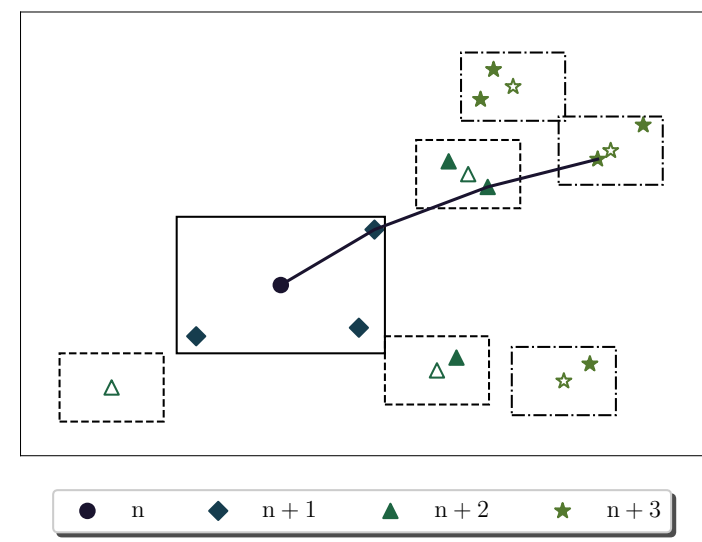

(b)

FIG. 1: Schematic comparison between tracking methods. Particle locations are denoted with filled symbols and predicted particle locations are denoted with hollow symbols.

(a) An example of the 4BE-NNI method. (b) An example of the 4BE-ETI method.

\section{DNS BENCHMARK OF TRACKING ALGORITHM}

\section{Dataset Description}

The performance (both in terms of tracking accuracy and efficiency) of the 4BE-ETI algorithm was analyzed and compared to the results from the traditional 4BE-NNI method using three-dimensional direct numerical simulations (DNS) available through the Johns Hopkins University Turbulence Databases ${ }^{31,32}$. Two datasets were explored: forced homogeneous isotropic turbulence and turbulent channel flow ${ }^{33}$.

The homogenous isotropic turbulence (HIT) dataset was selected to evaluate tracking when there was no mean flow. It is similar to both the DNS and the experimental datasets used to validate the original $4 \mathrm{BE}-\mathrm{NNI}$ tracking algorithm ${ }^{34}$. The domain for the DNS of isotropic turbulence was $2 \pi \times 2 \pi \times 2 \pi$, corresponding to a $1024^{3}$ spectral grid, and used periodic boundary conditions. The Taylor-scale Reynolds number is $R e_{\lambda}=\frac{u^{\prime} \lambda}{\nu}=418$. In 
contrast, the turbulent channel (Channel) dataset was selected to evaluate tracking when there was a strong mean flow that is strongly inhomogeneous. The turbulent channel DNS domain was $8 \pi \times 2 \times 3 \pi$, with periodic boundary conditions. The friction velocity Reynolds number was $R e_{\tau}=\frac{U_{c} h}{\nu}=10^{3}$.

To query the databases, the flow was initially seeded with 30,000 tracer particles for the HIT dataset and 50,000 tracer particles for the channel dataset throughout the entire volume. More particles were used for the channel dataset to maintain approximately constant particle number concentration, since the fluid domain volume was larger. The particles were then advected through the domain for each time step based on the resolved DNS flow field ${ }^{35}$. The trajectories were then sampled in a subdomain, creating a time sequence of particle locations as their trajectories entered and left the measurement volume, as is typical in experiments. The 4BE tracking method, both with traditional NNI and the proposed ETI, was applied to the particle positions, and the tracking results were compared to the ground-truth trajectories from the DNS datasets.

Several subsets of each dataset were generated by increasing the time between frames, thus varying the particle inter-frame displacements. The number of particles in each subset was kept constant. A wide range of values of the non-dimensional displacement-spacing ratio $\xi$, defined as the ratio of the average distance each particle moves between frames to the average separation between particles in a given frame ${ }^{13,19}$, is used in this benchmarking of the tracking methods to evaluate their domain of applicability in terms of maximum particle density and maximum particle displacement between frames. When $\xi$ is small, tracking is trivial because the particles move very little between frames and there are not many particles to consider for track continuation. However, as this non-dimensional displacement increases, tracking becomes more difficult because the particles move a large amount between frames and there are many particles that are candidates per frame to continue a track. The results of this study are shown over a wide range of $\xi$ values, from trivial to very challenging, in terms of tracking difficulty.

\section{Results}

The performance of the different initialization methods was evaluated by looking at the tracking error, defined as ${ }^{19}$ : 


$$
E_{\text {track }}=\frac{N_{\text {imperfect }}}{N_{\text {total }}}
$$

where $N_{\text {imperfect }}$ is the number of tracks that contain at least one incorrect particle position (and correspondingly velocity and acceleration), while $N_{\text {total }}$ is the total number of tracks in the dataset. A perfect track must start at the same point as the actual track and must contain no spurious locations. When $E_{\text {track }}$ is zero, the tracking code perfectly tracks all the tracks in the DNS dataset. When $E_{\text {track }}$ is close to or equal to one, the tracking code fails for almost every particle location, and most of the tracks in the dataset generated from the DNS are not recovered.

The results for the homogeneous isotropic turbulent flow are shown in Fig. 2. The Enhanced Track Initialization proposed here (4BE-ETI) performs better than the traditional nearest neighbor initialization (4BE-NNI) for this flow, where the mean velocity is zero and the inhomogeneity is low. For $\xi \lesssim 0.05$, the proposed $4 \mathrm{BE}-\mathrm{ETI}$ method has zero tracking error. For $0.05<\xi \lesssim 0.2$, the two methods follow a similar trend where the tracking error increases at approximately the same rate. For values frequently found in turbulent particleladen flow experiments ${ }^{36-39}$ where clustering is common, $0.2<\xi \lesssim 0.7$, the tracking error of the proposed 4BE-ETI method is half of the nearest neighbor's (4BE-NNI), extending the applicability of this multiframe 3D-PTV method. Finally, at very high $\xi$ values $(\xi \gtrsim 0.8)$, the error in the tracking reaches unacceptable values for both tracking methods tested. 


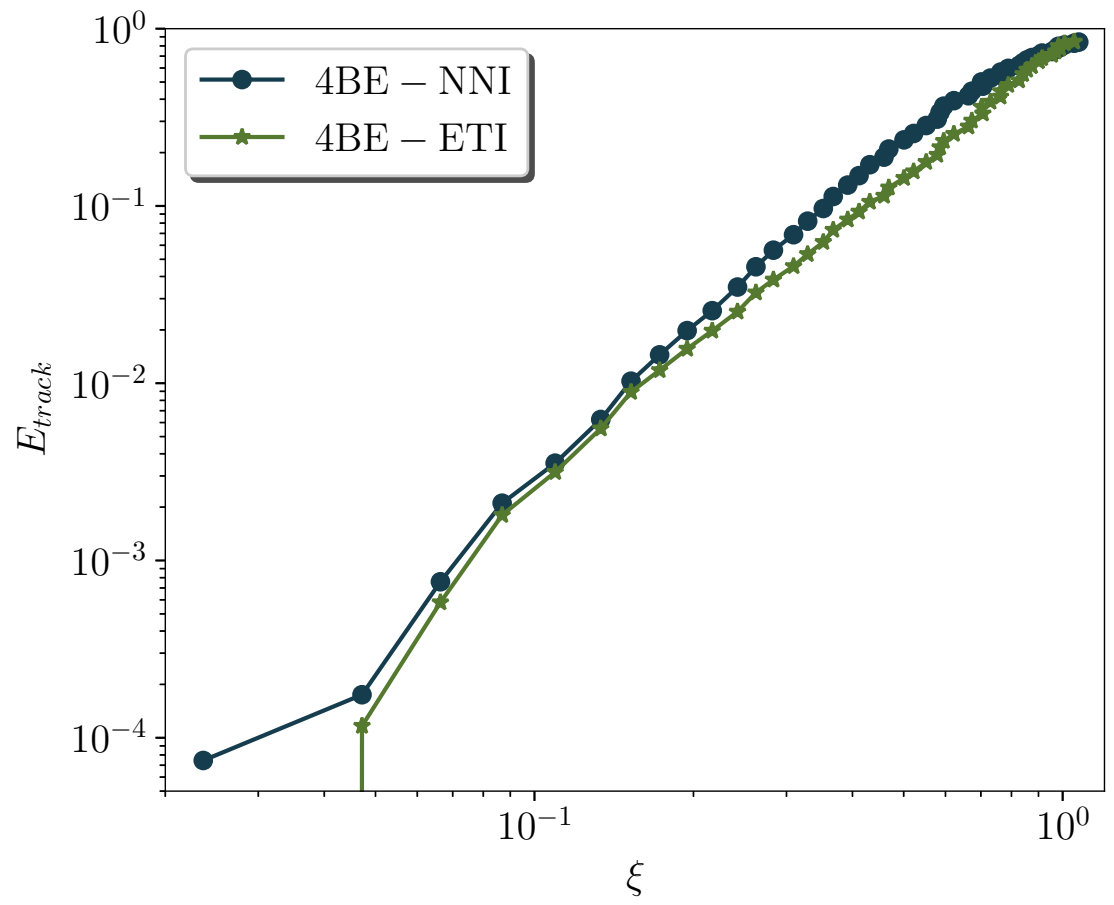

FIG. 2: Tracking performance of the 4BE-ETI method, compared to the baseline 4BE-NNI, in forced homogeneous isotropic turbulence. At values of $\xi<0.05$, the 4BE-ETI tracking error is zero.

Figure 3 shows the results of the tracking applied to the particles in turbulent channel flow. The 4BE-ETI performs significantly better than the 4BE-NNI for this anisotropic flow with spatially-variable non-zero mean velocity. For $\xi \lesssim 0.2$, there is zero tracking error when using 4BE-ETI method. For $\xi$ values $0.2<\xi \lesssim 0.7$, the tracking error is reduced significantly in the 4BE-ETI compared to the 4BE-NNI, up to an order of magnitude. Finally, at very high $\xi$ values $(\xi \gtrsim 0.7)$, where both methods failed in the HIT flow, the tracking error for the 4BE-ETI continues to be much smaller than for the 4BE-NNI, and has about 10-20\% of incorrect tracks even at these high values of $\xi$. This shows significant advantage of the Enhanced Track Initialization method for inhomogeneous and anisotropic flows where the error is significantly smaller and usage of the method is possible even at high $\xi$ values. 


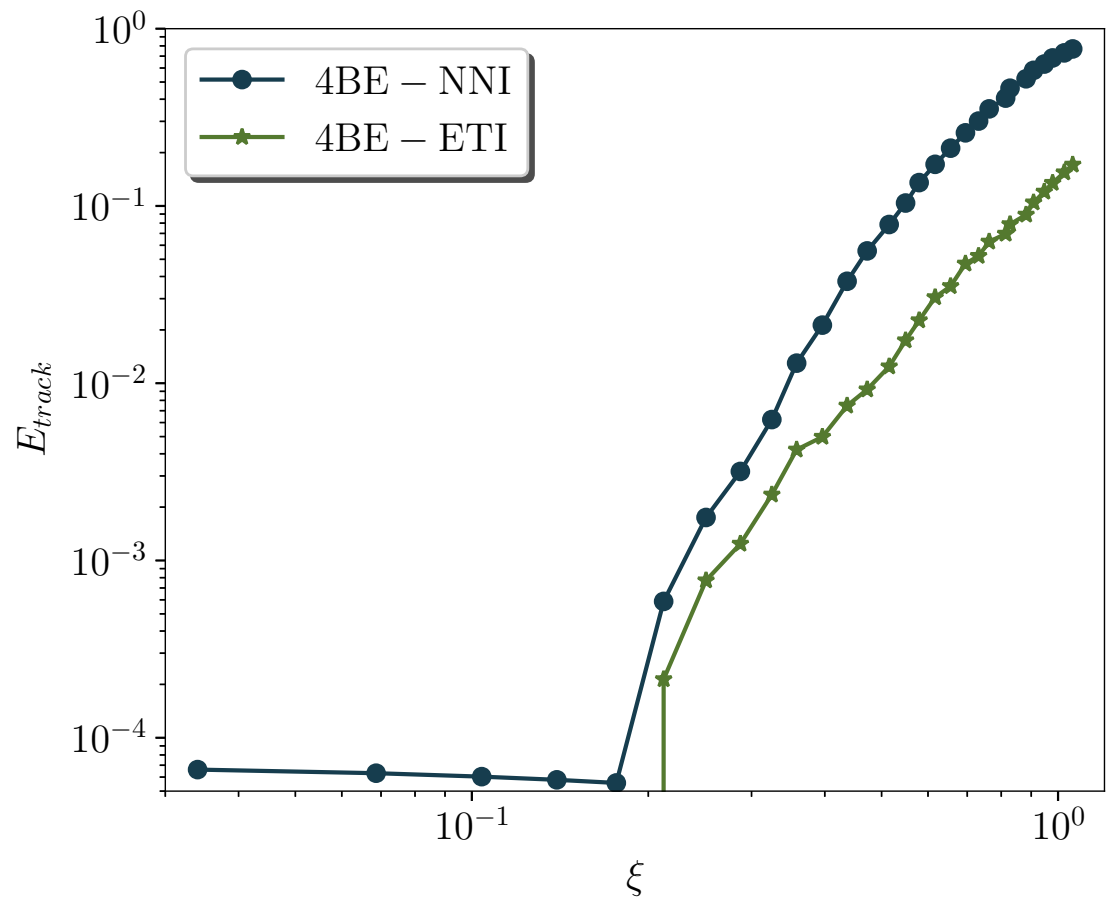

FIG. 3: Tracking performance of the 4BE-ETI method, compared to the baseline 4BE-NNI, in a turbulent channel flow. At values of $\xi<0.2$, the 4BE-ETI tracking error is zero.

However, from Figs. 2 and 3, we see that $\xi$ is an incomplete metric to compare tracking performance in different types of turbulent flows where the mean flow magnitudes differ and the levels of inhomogeneity and anisotropy vary from negligible to dominant. The 4BENNI tracking algorithm has a lower tracking error up to higher $\xi$ values in the turbulent channel than in the homogenous isotropic turbulence. This discrepancy is due to the fact that $\xi$ does not correctly consider turbulent fluctuations, or spatial inhomogeneity of the mean and fluctuating velocities. Therefore, we propose an additional metric $\xi^{\prime}$, defined as the ratio of the average displacement of a particle between frames due to turbulent fluctuations to the average separation between particles in a given frame, to more accurately compare the tracking in these two different types of turbulent flows. If there are strong turbulent fluctuations, tracking becomes more difficult because the velocity and acceleration predictions used to continue the tracks become less accurate. Like $\xi$, when $\xi^{\prime}$ is small, tracking is trivial because the particles move very little between frames and there are not many particles to consider for track continuation. However, as this ratio increases, tracking becomes more difficult because there are more turbulent fluctuations or there are more 
particles per image, or both. For $\xi^{\prime} \lesssim 0.2$, we see that the 4BE-NNI (HIT) and 4BE-ETI (both Channel and HIT) algorithms perform similarly and have tracking errors lower than 20\%. In the channel flow, the 4BE-NNI method performs worse than 4BE-ETI since the initialization fails in an inhomogeneous mean flow (even at smaller $\xi^{\prime}$, the performance of $4 \mathrm{BE}-\mathrm{NNI}$ is significantly worse than $4 \mathrm{BE}-\mathrm{ETI})$. At higher values of $\xi^{\prime}$, the results for the two datasets begin to diverge, which is most likely due to increased tracking difficulty at these high $\xi^{\prime}$ values, where the advantages of the novel 4BE-ETI are more pronounced.

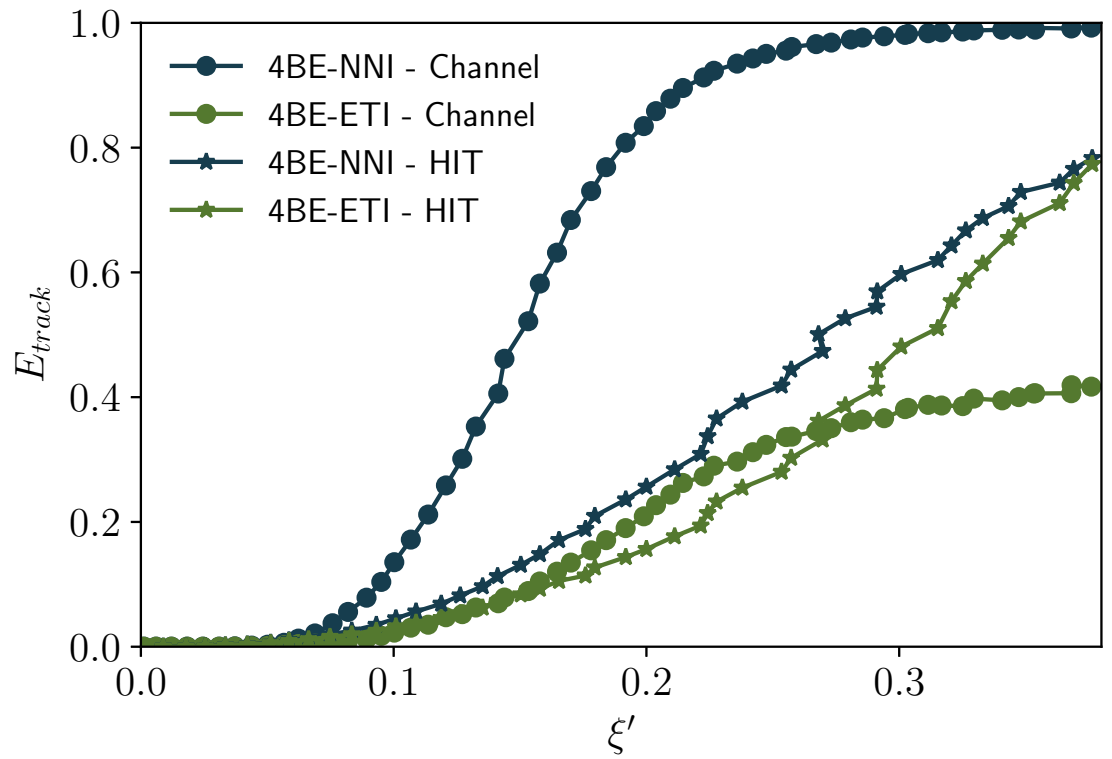

FIG. 4: Tracking performance of the 4BE-ETI method, compared to the baseline 4BE-NNI, in both forced homogeneous isotropic turbulence and a turbulent channel flow evaluated using $\xi^{\prime}$, based on turbulent fluctuations.

\section{CONCLUSION}

The method used for initialization of the particle tracks in multiframe 3D-PTV influences tracking performance strongly. We have shown that the Enhanced Tracking Initialization proposed allows the four-frame best estimate particle tracking method to perform significantly better than the nearest neighbor initialization. In turbulent channel flow, where the inhomogeneity and anisotropy of the flow presents a challenge to the nearest neighbor, 4BEETI has a tracking error up to an order of magnitude lower over a wide range of $\xi$ values, extending its applicability to densely seeded flows or flows where the inter-frame time is 
limited by the camera frame rate or laser repetition rate. The 4BE-ETI method proposed here also reduces error in tracking by approximately $50 \%$, with respect to the $4 \mathrm{BE}-\mathrm{NNI}$, in homogeneous isotropic turbulence. This highlights the flexibility of the 4BE-ETI to choose search region size and shape based on the flow characteristics to optimize accuracy and efficiency without undue increases in computational time. Additionally, automated strategies can be easily implemented by running the tracking on a small subset of particles, equally spaced along the flow domain, with a large initialization region to obtain coarse information about the flow and recursively using this information to refine the initial search region geometry in an increasing number of particles for subsequent passes.

\section{ACKNOWLEDGMENTS}

This work was facilitated though the use of advanced computational, storage, and networking infrastructure provided by the Hyak supercomputer system at the University of Washington. This work was sponsored by the Office of Naval Research (ONR), as part of the Multidisciplinary University Research Initiatives (MURI) Program, under grant number N00014-16-1-2617. The views and conclusions contained herein are those of the authors only and should not be interpreted as representing those of ONR, the U.S. Navy or the U.S. Government. The authors also wish to thank Stephanie Paustian Villegas and Colin Bateson for discussions on particle tracking.

\section{REFERENCES}

${ }^{1}$ F. Toschi and E. Bodenschatz, "Lagrangian properties of particles in turbulence," Annual Review of Fluid Mechanics, vol. 41, pp. 375-404, 2009.

${ }^{2}$ G. A. Voth and A. Soldati, "Anisotropic particles in turbulence," Annual Review of Fluid Mechanics, vol. 49, pp. 249-276, 2017.

${ }^{3}$ N. Machicoane, M. López-Caballero, M. Bourgoin, A. Aliseda, and R. Volk, "A multitime-step noise reduction method for measuring velocity statistics from particle tracking velocimetry," Measurement Science and Technology, vol. 28, no. 10, p. 107002, 2017. 
${ }^{4}$ R. G. Racca and J. M. Dewey, "Automatic particle tracking in a three-dimensional flow field," in 16th Intl Congress on High Speed Photography and Photonics, vol. 491. International Society for Optics and Photonics, 1985, pp. 864-872.

${ }^{5}$ R. Racca and J. Dewey, "A method for automatic particle tracking in a three-dimensional flow field," Experiments in Fluids, vol. 6, no. 1, pp. 25-32, 1988.

${ }^{6}$ T. Kobayashi, T. Saga, and K. Sekimoto, "Velocity measurement of three-dimensional flow around rotating parallel disks by digital image processing," in Scatter from optical components, 1989.

${ }^{7}$ K. Nishino, N. Kasagi, and M. Hirata, "Three-dimensional particle tracking velocimetry based on automated digital image processing," Journal of Fluids Engineering, vol. 111, pp. 384-391, 1989.

${ }^{8}$ D. Papantoniou and T. Dracos, "Analyzing 3-d turbulent motions in open channel flow by use of stereoscopy and particle tracking," in Advances in Turbulence 2, H. Fernholz and H. Fiedler, Eds. Berlin, Heidelberg: Springer, 1989, pp. 278-285.

${ }^{9}$ Y. Sata, K. Nishino, and N. Kasagi, "Whole field measurement of turbulent flows using a three-dimensional particle tracking velocimeter," in Flow Visualization V; Proceedings of the 5th International Symposium, 1990, pp. 248-253.

${ }^{10}$ Y. Hassan and R. Canaan, "Full-field bubbly flow velocity measurements using a multiframe particle tracking technique," Experiments in Fluids, vol. 12, pp. 49-60, 1991.

${ }^{11}$ N. Kasagi and K. Nishino, "Probing turbulence with three-dimensional particle-tracking velocimetry," Experimental Thermal and Fluid Science, vol. 4, pp. 601-612, 1991.

${ }^{12}$ T. Kobayashi, T. Saga, and S. Segawa, "Multipoint velocity measurements for unsteady flow field by digital image processing," in Flow Visualization VI; Proceedings of the 6th International Symposium, 1992.

${ }^{13}$ N. A. Malik, T. Dracos, and D. A. Papantoniou, "Particle tracking velocimetry in threedimensional flows part ii: Particle tracking," Experiments in Fluids, vol. 15, pp. 279-294, 1993.

${ }^{14}$ Y. Guezennec, R. Brodkey, N. Trigui, and J. Kent, "Algorithms for fully automated threedimensional particle tracking velocimetry," Experiments in Fluids, vol. 17, pp. 209-219, 1994. 
${ }^{15}$ T. Dracos, "Particle tracking in three-dimensional space," in Three-Dimensional Velocity and Vorticity Measuring and Image Analysis Techniques, T. Dracos, Ed. Dordrecht: Springer, 1996, vol. 4, pp. 209-227.

${ }^{16}$ H. Stüer, H.-G. Maas, M. Virant, and J. Becker, "A volumetric 3d measurement tool for velocity field diagnostics in microgravity experiments," Measurement Science and Technology, vol. 10, pp. 904-913, 1999.

${ }^{17}$ K. Ohmi and H.-Y. Li, "Particle-tracking velocimetry with new algorithms," Measurement Science and Technology, vol. 11, p. 603-616, 2000.

${ }^{18}$ J. Willneff, "A spatio-temporal matching algorithm for $3 \mathrm{~d}$ particle tracking velocimetry," Ph.D. dissertation, Swiss Federal Institute of Technology Zurich, 2003.

${ }^{19}$ N. T. Ouellette, H. Xu, and E. Bodenschatz, "A quantitative study of three-dimensional lagrangian particle tracking algorithms," Experiments in Fluids, vol. 40, no. 2, pp. 301-313, 2006.

${ }^{20}$ D. Li, Y. Zhang, Y. Sun, and W. Yan, "A multi-frame particle tracking algorithm robust against input noise," Measurement Science and Technology, vol. 29, p. 105401, 2008.

${ }^{21}$ C. Cierpka, B. Lütke, and C. J. Kähler, "Higher order multi-frame particle tracking velocimetry," Experiments in Fluids, vol. 54, p. 1533, 2013.

22 "Open source particle tracking velocimetry - openptv," https://github.com/OpenPTV.

${ }^{23}$ D. Schanz, S. Gesemann, and A. Schröder, "Shake-the-box: Lagrangian particle tracking at high particle image densities," Experiments in Fluids, vol. 57, p. 70, 2016.

${ }^{24}$ H. Xu, M. Bourgoin, N. T. Ouellette, and E. Bodenschatz, "High order lagrangian velocity statistics in turbulence," Physics Review Letters, vol. 96, p. 024503, 2006.

${ }^{25}$ R. Bordás, C. Roloff, D. Thévenin, and R. Shaw, "Experimental determination of droplet collision rates in turbulence," New Journal of Physics, vol. 15, p. 045010, 2013.

${ }^{26}$ R. Ni, S. Kramel, N. T. Ouellette, and G. A. Voth, "Measurements of the coupling between the tumbling of rods and the velocity gradient tensor in turbulence," Journal of Fluid Mechanics, vol. 766, pp. 202-225, 2015.

${ }^{27}$ M. Cisse, E. Saw, M. Gibert, E. Bodenschatz, and J. Bec, "Turbulence attenuation by large neutrally buoyant particles," Physics of Fluids, vol. 27, p. 061702, 2015.

${ }^{28}$ B. C. Cole, G. G. Marcus, S. Parsa, S. Kramel, R. Ni, and G. A. Voth, "Methods for measuring the orientation and rotation rate of 3d-printed particles in turbulence," Journal of Visualized Experiments, vol. 112, p. 53599, 2016. 
${ }^{29}$ N. Stelzenmuller, J. I. Polanco, L. Vignal, I. Vinkovic, and N. Mordant, "Lagrangian acceleration statistics in a turbulent channel flow," Physical Review Fluids, vol. 2, p. $054602,2017$.

${ }^{30}$ J. M. Lawson, E. Bodenschatz, C. C. Lalescu, and M. Wilczek, "Bias in particle tracking acceleration measurement," Experiments in Fluids, vol. 59, p. 172, Nov. 2018.

${ }^{31}$ Y. Li, E. Perlman, M. Wan, Y. Yang, C. Meneveau, R. Burns, S. Chen, A. Szalay, and G. Eyink, "A public turbulence database cluster and applications to study lagrangian evolution of velocity increments in turbulence," Journal of Turbulence, vol. 9, no. 31, pp. $1-29,2008$.

${ }^{32}$ E. Perlman, R. Burns, Y. Li, and C. Meneveau, "Data exploration of turbulence simulations using a database cluster," in SC '07: Proceedings of the 2007 ACM/IEEE Conference on Supercomputing, November 2007, pp. 1-11.

${ }^{33}$ J. Graham, K. Kanov, X. I. A. Yang, M. Lee, N. Malaya, C. C. Lalescu, R. Burns, G. Eyink, A. Szalay, R. D. Moser, and C. Meneveau, "A web services accessible database of turbulent channel flow and its use for testing a new integral wall model for les," Journal of Turbulence, vol. 17, no. 2, pp. 181-215, 2016.

${ }^{34}$ G. A. Voth, A. L. Porta, A. M. Crawford, J. Alexander, and E. Bodenschatz, "Measurement of particle accelerations in fully developed turbulence," Journal of Fluid Mechanics, vol. 469, p. 121-160, 2002.

${ }^{35}$ H. Yu, K. Kanov, E. Perlman, J. Graham, E. Frederix, R. Burns, A. Szalay, G. Eyink, and C. Meneveau, "Studying lagrangian dynamics of turbulence using on-demand fluid particle tracking in a public turbulence database," Journal of Turbulence, vol. 13, p. 12, 2012 .

${ }^{36}$ A. Aliseda, A. Cartellier, F. Hainaux, and J. Lasheras, "Effect of preferential concentration on the settling velocity of heavy particles in homogeneous isotropic turbulence," Journal of Fluid Mechanics, vol. 468, pp. 77-105, 2002.

${ }^{37}$ A. Aliseda and J. Lasheras, "Effect of buoyancy on the dynamics of a turbulent boundary layer laden with microbubbles," Journal of Fluid Mechanics, vol. 559, pp. 307-334, 2006.

${ }^{38}$ C. P. Bateson and A. Aliseda, "Wind tunnel measurements of the preferential concentration of inertial droplets in homogeneous isotropic turbulence," Experiments in Fluids, vol. 52, pp. 1373-1387, 2012. 
${ }^{39}$ P. Huck, C. Bateson, R. Volk, A. Cartellier, M. Bourgoin, and A. Aliseda, "The role of collective effects on settling velocity enhancement for inertial particles in turbulence," Journal of Fluid Mechanics, vol. 846, pp. 1059-1075, 2018. 